\section{Zur Erforderlichkeit der Personenidentität bei einem Zulassungsverzicht zu Gunsten eines MVZ zwecks Anstellung}

\section{Einführung}

Die vertragsärztliche Versorgung unterliegt einem fortwährenden Strukturwandel. Immer mehr Ärzte entscheiden sich gegen die Selbständigkeit und nehmen die Möglichkeit der Anstellung wahr. Dieser Trend ist im Fachgebiet der Radiologie besonders stark ausgeprägt, wie die Zahlen der KBV belegen. In dem Zeitraum von 2007 bis 2016 sank die Zahl der niedergelassenen radiologischen Vertragsärzte von 2326 auf 1903. Dies entspricht einem Rückgang von 18,2\%. Im gleichen Zeitraum stieg die Anzahl der angestellten Radiologen in Einrichtungen von 155 auf 755 sowie in freien Praxen von 123 auf 706. Ihr Anteil erhöhte sich mithin um $400 \%$ bzw. um $474 \%$. Die Gründe für diese Entwicklung sind mannigfaltig. Neben der Scheu vor wirtschaftlichen Risiken zählen sicherlich auch der Wunsch nach geregelten Arbeitszeiten und damit einhergehend nach der besseren Vereinbarkeit von Beruf und Familie zu den Hauptgründen. Die Möglichkeit der Anstellung wird speziell im Rahmen eines MVZ jedoch auch dazu genutzt, um einen Wechsel in der Person des Arztes zu vollziehen. Dementsprechend ist das Gestaltungsmodell des Zulassungsverzichtes zu Gunsten der (eigenen) Anstellung im MVZ gem. §103 Abs. 4a S. 1 SGB V von großer praktischer Relevanz.

Um niederlassungswilligen Ärzten andererseits den Einstieg in die Freiberuflichkeit zu erleichtern, sieht § 103 Abs. 4a S. 2 SGB V die Möglichkeit vor, dass ein Arzt, dessen Sitz in einem gesperrten Planungsbereich liegt, nach einer Tätigkeit von mindestens fünf Jahren in einem MVZ unbeschadet der Zulassungsbeschränkungen auf Antrag eine Zulassung in diesem Planungsbereich erhält.

Allerdings dürfen beide Gestaltungsmodelle nicht dazu missbraucht werden, um die gesetzlichen Vorgaben, insbesondere die Regelungen des Nachbesetzungsverfahrens (§ 103 Abs. 3a S. 3 SGB V), zu umgehen. In den Vordergrund rückt daher Frage, ob und in welchen Grenzen mit dem Verzicht zwecks Anstellung bzw. dem Anspruch auf bedarfsunabhängige Zulassung nach fünf Jahren ein Personenwechsel einhergehen darf. Mit anderen Worten: Ist eine Personenidentität in der jeweiligen Fallkonstellation von Gesetzes wegen erforderlich? Eine aktuelle Entscheidung des Landessozialgerichts Nordrhein-Westfalen (LSG NRW, Urt.v. 3.5.2017, Az.: L 11 KA 76/14) greift diese Fragen auf. Zum einen hatte der Senat über die Auslegung der einzelnen Tatbestandsmerkmale des $§ 103$ Abs. 4a S. 2 SGB V zu befinden. Der streitgegenständliche Fall wies jedoch die Besonderheit auf, dass die Anstellungsgenehmigung einem früher erklärten Zulassungsverzicht entstammte. Folglich musste sich das Gericht zum anderen inzident auch mit der Frage nach der Rechtmäßigkeit des Zulassungsverzichtes gem. § 103 Abs. 4a S. 1 SGB V befassen.

\section{Sachverhalt}

Im Jahre 2005 wurde unter Beteiligung eines Universitätsklinikums ein MVZ gegründet, in welchem ein Facharzt für Radiologie (R) sowie eine Fachärztin für Strahlentherapie (S), die bis dato jeweils in eigener vertragsärztlicher Praxis tätig waren, sowie ein an der Klinik beschäftigter Facharzt für Diagnostische Radiologie (K) angestellt wurden. Wegen bestehender Zulassungsbeschränkungen verzichtete die Fachärztin für Strahlentherapie (S) auf ihre (volle) Zulassung als Vertragsärztin zu Gunsten ihrer (vollschichtigen) Anstellung beim MVZ. Ebenso verzichtete der Facharzt für Radiologie ( $R$ ) auf seine Zulassung zu Gunsten des MVZ, um jedoch nicht vollschichtig, sondern nur in einem Umfang von 10 Stunden die Woche für das MVZ tätig werden. Die dadurch entstandene Vakanz i.H.v. 30 Wochenstunden füllte der bis dahin nicht an der vertragsärztlichen Versorgung teilnehmende Krankenhausarzt (K) zu einer Vollzeitstelle auf und wurde somit faktisch auf der von seinem Kollegen „eingebrachten“ Stelle (mit-) tätig.

Im darauffolgenden Jahr beendete der Facharzt für Radiologie ( $R$ ) seine Tätigkeit für das MVZ. Der Facharzt für Diagnostische Radio-

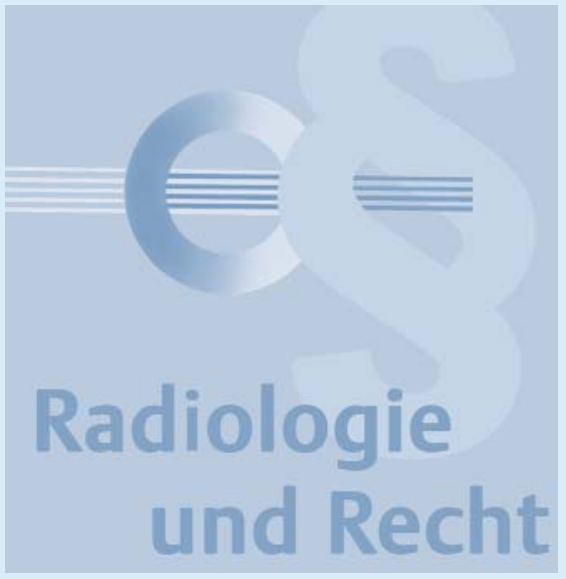

logie (K) übernahm dessen Wochenstunden und arbeitete fortan in Vollzeit. Zwischenzeitlich wurde außerdem die Rechtsform des MVZ in eine $\mathrm{GmbH}$ geändert.

Unter Berufung auf § 103 Abs. 4a S. 2 SGB V begehrte der Facharzt für Diagnostische Radiologie (K) im Jahr 2011 schließlich eine eigene Zulassung. Sein Antrag wurde jedoch sowohl vom Zulassungs- als auch vom Berufungsausschuss mit der Begründung abgelehnt, dass er auf Grund einer Nachbesetzung tätig geworden sei und daher nicht in den Genuss der privilegierenden Regelung kommen dürfe.

\section{Rechtliche Würdigung}

Die hiergegen gerichtete Klage hatte in der Sache Erfolg. Diesem Standpunkt schloss sich das LSG NRW an und wies die Berufung als unbegründet zurück. Angesichts der Komplexität der Rechtsmaterie wird der streitgegenständliche Fall - entgegen den Urteilsgründen - nicht mittels einer Inzidentprüfung, sondern in chronologischer Reihenfolge gewürdigt.

\section{Zulassungsverzicht zu Gunsten eines MVZ zwecks Anstellung}

Gem. §95 Abs. 2 S. 7 SGB V, §32b Abs. 2 S. 1i. V.m. $\S 1$ Abs. 3 Nr. 2 Ärzte-ZV bedarf die Anstellung eines Arztes in einem MVZ der Genehmigung des Zulassungsausschusses. Die Genehmigung ist nach $\S 95$ Abs. 2 S. 9 SGB $V$ abzulehnen, wenn bei Antragstellung wegen Überversorgung für die dort tätigen Ärzte Zulassungsbeschränkungen nach § 103 Abs. 1 S. 2 SGB $V$ angeordnet worden sind. Als Ausnahme von diesem Grundsatz gestattet das Gesetz (lediglich) 
die Anstellung im Wege der Nachbesetzung einer Arztstelle in einem MVZ gem. $§ 103$ Abs. 4a S. 3 SGB V. Dies erfordert jedoch die Durchführung eines formellen Nachbesetzungsverfahrens, welches im streitgegenständlichen Fall nicht erfolgt ist. Vielmehr kommt das Bundessozialgericht (BSG) in einer Entscheidung vom 4.5.2016 (Az. B 6 KA 21/15 R), auf welches das LSG NRW Bezug nimmt, zu dem Schluss:

„Gegen eine Auslegung des $\S 103$ Abs. 4a S. 1 und 3 SGB V dahin, dass dem MVZ [selbst] ein Recht zur Nachbesetzung im Umfang der Zulassung zuwachsen würde, auf die der in das MVZ wechselnde Arzt mit dem Ziel der Anstellung verzichtet hat, sprechen auch systematische Gründe: Verzichtet ein Arzt in einem wegen Überversorgung gesperrten Planungsbereich auf seine Zulassung, stehen ihm drei [abschließende] Gestaltungsmöglichkeiten zur Verfügung. Zunächst kann er es bei der Erklärung des Verzichts auf die Zulassung bewenden lassen. Dies hat zur Folge, dass die Zulassung ersatzlos entfällt und sich der Grad der (Über-) Versorgung entsprechend reduziert.

Der Vertragsarzt kann weiterhin den Verzicht auf die Zulassung mit einem Antrag auf Durchführung eines Verfahrens zur Nachbesetzung des Praxissitzes verbinden. Wenn sich nach positiver Entscheidung gemäß §103 Abs. 3a Satz 1 SGB V über die Durchführung eines Nachbesetzungsverfahrens mehrere Ärzte um die Praxisnachfolge bewerben, sieht $\$ 103$ Abs. 4 SGB V eine Auswahlentscheidung des Zulassungsausschusses vor. Um die Praxisnachfolge kann sich auch ein MVZ bewerben. $\S 103$ Abs. 4c Satz 1 SGB V [...] sieht ausdrücklich die Möglichkeit einer Weiterführung der Praxis in der Form vor, dass ein MVZ den Vertragsarztsitz übernimmt und die vertragsärztliche Tätigkeit durch einen angestellten Arzt in der Einrichtung weiterführt. [...] Die Auswahl des angestellten Arztes obliegt in diesem Fall zwar dem MVZ [...]. Die Entscheidung, ob das MVZ [...] die Nachfolge antritt oder ob ein anderer Bewerber um die Nachfolge ausgewählt wird, trifft jedoch der Zulassungsausschuss.

Eine dritte Möglichkeit besteht darin, dass der Vertragsarzt, der auf seine Zulassung verzichtet, selbst als Angestellter [...] in einem MVZ tätig wird. Für diesen Fall ist weder eine Entscheidung des Zulassungsausschusses zum ,Ob' der Nachbesetzung noch eine Auswahlentscheidung zwischen mehreren Bewerbern um die Praxisnachfolge vorgesehen. Vielmehr ist dem MVZ die Genehmigung zur Anstellung des Arztes zu erteilen, der auf seine Zulassung verzichtet hat. Entscheidende Voraussetzung für diese Privilegierung ist nach § 103 Abs. 4a Satz 1 SGB V, dass der Arzt auf seine Zulassung verzichtet hat, ,um in einem medizinischen Versorgungszentrum tätig zu werden: “

Gestützt werde dieses Umgehungsverbot, so das BSG, durch die gesetzgeberischen Wertungen im Rahmen des GKV-Versorgungsstärkungsgesetzes vom 16.7.2015 (BGBI. I, S. 1211), die in den erweiterten Möglichkeiten zum Abbau einer bestehenden Überversorgung zum Ausdruck kommen. Dementsprechend wird die Entscheidung über die Nachbesetzung eines Vertragsarztsitzes bei einer Überschreitung des allgemeinen bedarfsgerechten Versorgungsgrades um mindestens $40 \%$ für den Regelfall nicht mehr in das Ermessen der Zulassungsgremien gestellt. Vielmehr soll der Zulassungsausschuss den Antrag auf Durchführung des Nachbesetzungsverfahrens gem. §103 Abs. 3a S. 7, Abs. 1 S. 3 SGB V in diesem Fall ablehnen, wenn eine Nachbesetzung aus Versorgungsgründen nicht erforderlich ist.

Daraus folgt, dass ein MVZ die Privilegien, die § 103 Abs. 4a SGB V im Rahmen der Nachbesetzung vermittelt, grundsätzlich nur in Anspruch nehmen kann, wenn und soweit der Arzt auf seine Zulassung gerade mit dem Ziel verzichtet, selbst in dem MVZ als angestellter Arzt tätig zu werden. Es ist mithin eine Personenidentität zwischen dem auf die Zulassung verzichtenden Arzt und dem Arzt, der die Anstellung in dem MVZ aufnimmt, erforderlich. Wenn die Stelle eines ehemaligen Vertragsarztes, der seine Tätigkeit als Angestellter im MVZ von Anfang an nur im Umfang einer Teilzeittätigkeit aufgenommen hat, gleichwohl mit einer vollen Stelle nachbesetzt werden könnte, würde darin eine Umgehung der für die Nachfolgezulassung geltenden Voraussetzungen liegen. Das MVZ könnten die Stelle hinsichtlich des überschießenden Anteils erstmals mit einem selbst ausgewählten „Nachfolger“ besetzen. Diese Möglichkeit sieht § 103 Abs. 4a S. 1 SGB V jedoch gerade nicht vor. Die Anstellung eines anderen Arztes als desjenigen, der auf die Zulassung verzichtet hat, ist nicht Gegenstand des $§ 103$ Abs. 4a S. 1 SGB V, sondern des $\S 103$ Abs. 4c SGB V, der eine Auswahlentscheidung der Zulassungsgremien verlangt.
Zur Dokumentation des Willens „Verzicht, um in einem MVZ tätig zu werden“ verlangt das BSG grundsätzlich eine mindestens dreijährige Tätigkeit als angestellter Arzt. Erst nach Ablauf dieses Zeitraumes könne davon ausgegangen werden, dass der Verzicht zwecks Anstellung auch tatsächlich gewollt und gelebt worden ist. Gleichwohl gestattet das Gericht eine jährliche Reduktion des Beschäftigungsumfangs um den Anrechnungsfaktor 0,25.

Bezogen auf den streitgegenständlich Fall bedeutet dies, dass die Anstellung des Facharztes für Diagnostische Radiologie (K) mit einem Volumen von 30 Wochenstunden mangels Personenidentität sowie mangels fehlender Durchführung eines Nachbesetzungsverfahrens in rechtswidriger Weise erfolgt und damit die Genehmigung des Zulassungsausschusses zu Unrecht ergangen ist. Auf Grund von Vertrauensschutzerwägungen sowie der Bestandskraft der Genehmigung des Zulassungsausschusses wirkte sich die Rechtswidrigkeit auf das weitere Verfahren jedoch nicht aus. Folglich muss die Anstellung des Arztes im Rahmen seines Antrags auf Zulassung als rechtmäßig erfolgt angesehen werden.

\section{Anspruch auf bedarfsunabhängige Zulassung nach fünf Jahren}

Anspruchsgrundlage für das Begehren einer bedarfsunabhängigen Zulassung ist $\S 103$ Abs. 4a S. 2 SGB V. Demgemäß erhält ein Arzt „nach einer Tätigkeit von mindestens fünf Jahren in einem medizinischen Versorgungszentrum, dessen Sitz in einem Planungsbereich liegt, für den Zulassungsbeschränkungen angeordnet sind, [...] unbeschadet der Zulassungsbeschränkungen auf Antrag eine Zulassung in diesem Planungsbereich; dies gilt nicht für Ärzte, die auf Grund einer Nachbesetzung nach Satz 5 [Redaktionsversehen des Gesetzgebers, gemeint ist Satz 3] oder erst seit dem 1. Januar 2007 in einem medizinischen Versorgungszentrum tätig sind." Nach Auffassung des erkennenden Senates waren diese Voraussetzungen im vorliegenden Fall erfüllt. Problematisch waren jedoch drei Aspekte.

Zum einen stellte sich die Frage, ob der zwischenzeitliche Rechtsformwechsel Einfluss auf das Erfordernis der mindestens fünfjährigen Tätigkeit im MVZ hat. Dies verneinte das 
Gericht. Weder dem Gesetzeswortlaut noch der Gesetzessystematik sind Anhaltspunkte dafür zu entnehmen, dass der bloße Rechtsformwechsel eines MVZ bei sonst identisch fortbestehender Tätigkeit des MVZ sowie der angestellten Ärzte die Privilegierung nach § 103 Abs. 4a S. 2 SGB V ausschließen soll. Vielmehr geht aus dem Wortlaut der Norm und den Gesetzesmaterialien hervor, dass das wesentliche Kriterium für die privilegierte Zulassung sein soll, ob der betroffene Arzt durch seine Anstellung am MVZ „dessen Gründung oder die Erweiterung dessen ärztlichen Behandlungsangebots ermöglicht hat", oder erst später im Wege einer „Nachbesetzung“ für das MVZ tätig geworden ist (BT-Drs. 15/1525, S. 112).

Problematisch war zum anderen die fehlende Personenidentität von dem antragsstellenden Arzt und dem Arzt, welcher die Zulassung in das MVZ eingebracht hat. Im Gegensatz zu § 103 Abs. 4a S. 1 SGB V ist in diesem Kontext ein Personenwechsel allerdings unschädlich. Im Rahmen des $\S 103$ Abs. 4a S. 2 SGB V muss es sich nicht um einen Arzt handeln, der vor seiner Tätigkeit für das MVZ nach Satz 1 der Norm auf seine Zulassung zur vertragsärztlichen Versorgung zu Gunsten einer Tätigkeit im MVZ verzichtet hat. Gegen ein solches Verständnis der Vorschrift spricht bereits ihr Wortlaut. So ist in Satz 2 von „einem“ „Arzt“ die Rede, in Satz 1 hingegen von einem „Vertragsarzt“. Auf Satz 1 wird in Satz 2 auch nicht dadurch Bezug genommen, dass von „diesem Arzt“, „demselben Arzt“ o. ä. gesprochen wird. Stattdessen wird ein unbestimmter Artikel verwendet. Die Gesetzesmaterialien bestätigen die fehlende Inbezugnahme (BT-Drs. 15/1525, S. 112):

„Diese besondere Niederlassungsmöglichkeit erhöht die Attraktivität des medizinischen Versorgungszentrums für junge Ärzte, da diese durch eine fünfjährige Tätigkeit als angestellte Ärzte einer derartigen Einrichtung nicht nur Erfahrungen für eine spätere freiberufliche Tätigkeit sammeln, sondern aufgrund dieser Regelung auch die Möglichkeit erhalten, in einem gesperrten Gebiet in die Freiberuflichkeit zu wechseln, ohne den normalerweise notwendigen Weg über die Praxisübergabe nach $\S 103$ Abs. 4 SGB V gehen zu müssen. “

Es geht infolgedessen nicht um denselben Vertragsarzt, der zuvor bereits freiberuflich tätig war und dann auf seine Zulassung zu Gunsten einer Tätigkeit im MVZ verzichtet hat, da solche Ärzte in einem MVZ keine „Erfahrungen für eine spätere freiberufliche Tätigkeit sammeln" müssen (LSG Berlin-Brandenburg, Urt. v. 26.2.2016, Az. L 24 KA 68/14).

Fraglich war nicht zuletzt, ob der antragsstellende Arzt im Wege einer Nachbesetzung nach Satz 3 (1. Ausschlusstatbestand) tätig geworden ist. Der Begriff des Nachbesetzens in $\S 103$ Abs. 4a S. 2 und 5 SGB V ist gesetzlich nicht definiert. Ausgehend vom allgemeinen Sprachgebrauch bedeutet er „einen frei werdenden Posten wieder neu besetzen“.

Das MVZ ist mit Wirkung zum 1.11.2005 gegründet worden. Alle damals vom MVZ angestellten Ärzte sind erstmals angestellt worden. Demzufolge gab es zu diesem Zeitpunkt noch keinen bereits einmal vergebenen, dann jedoch freigewordenen „Posten“ (Arztstelle), den das MVZ hätte wieder neu besetzen können. Eine Nachbesetzung liegt somit nicht vor. Es darf nach Meinung des Gerichtes zudem nicht darauf abgestellt werden, dass die Erstbesetzung der Stelle des Facharztes für Radiologie (K) nur durch den vorausgegangenen Zulassungsverzicht eines anderen Arztes möglich war. Zum einen erfolgte die Zulassung als Vertragsarzt in eigener Praxis nicht in Form einer (Erst-) Besetzung durch das MVZ. Das wäre aber Voraussetzung dafür, dass das MVZ anschließend die Stelle mit dem Facharzt für Radiologie (K) „nachbesetzt" hat, so der eindeutige Wortlaut des $\S 103$ Abs. 4a S. 2, 2. HS und S. 5 SGB V. Zum anderen werden von $\S 103$ Abs. 4a S. 2 SGB V allein die Fälle erfasst, in denen Zulassungsbeschränkungen bestehen. Die von einem MVZ in einem solchen Fall zu besetzenden Stellen entstehen nicht aus dem Nichts, werden also nicht originär geschaffen, sondern sind zuvor stets von einem anderen (Vertrags-) Arzt ausgefüllt worden. Wenn bereits dies eine „Nachbesetzung“ im Sinne des §103 Abs. 4a S. 2 SGB V ausschließen würde, liefe die Vorschrift leer, da es denklogisch nur „Nachbesetzungen“ gäbe.

Zu demselben Ergebnis führt die historischen Auslegung auf Grundlage der Gesetzesmaterialien (BT-Drs. 15/1525, S. 112):

"[Satz 2] erlaubt angestellten Ärzten eines Medizinischen Versorgungszentrums, die durch ihre Anstellung in einem Medizinischen Versor- gungszentrum dessen Gründung oder die Erweiterung dessen ärztlichen Behandlungsangebots ermöglicht haben, nach mindestens fünf Jahren in dem betreffenden Planungsbereich auch dann in die Niederlassung zu wechseln, wenn dieser Planungsbereich wegen überversorgung gesperrt ist. “

Nach der Gesetzesbegründung sollte mithin nur derjenige angestellte Arzt, der lediglich in einem MVZ auf eine bereits bestehende Arztstelle nachbesetzt wird, nicht von der Privilegierung des $\S 103$ Abs. 4a S. 2 SGB V profitieren. Hingegen sollten die Ärzte, die - wie im streitgegenständlichen Fall - bereits bei der „Gründung“ eines MVZ bei diesem angestellt waren und so die „Gründung oder Erweiterung dessen ärztlichen Behandlungsangebots ermöglicht haben“, privilegiert werden.

\section{Fazit}

Die Entscheidung des LSG NRW folgt in ihrem ersten Teil betreffend den Zulassungsverzicht zwecks Anstellung ( $\$ 103$ Abs. 4a S. 1 SGB V) konsequent der Judikatur des BSG. Auf Grund des statuierten Erfordernisses der Personenidentität werden zwar die Gestaltungsmöglichkeiten eingeschränkt. Die Rechtsprechungslinie stimmt jedoch hinsichtlich ihres Grundgedanken - mit den gesetzlichen Wertungen überein und fordert daher dogmatisch zutreffend eine Personenidentität. Ebenso kommt das Gericht im zweiten Teil der Urteilsbegründung in rechtlich nicht zu beanstandender Weise zu dem Schluss, dass der Anspruch auf eine bedarfsunabhängige Zulassung nach fünf Jahren (§103 Abs. 4a S. 2 SGB V) keine Personenidentität voraussetzt.

Prof. Dr. Peter Wigge

Rechtsanwalt

Fachanwalt für Medizinrecht

Dipl. jur. Jan Harald Schütz, LL.M., Wiss. Mitarbeiter

Rechtsanwälte Wigge

Scharnhorststraße 40

48151 Münster

Telefon: (0251) 53595 - 0

Telefax: (0251) 53595 - 99

E-Mail: kanzlei@ra-wigge.de

www.ra-wigge.de 